\title{
Interactive comment on "Evaluation of WRF-DART (ARW v.3.9.1.1 and DART manhattan release) multi-phase cloud water path assimilation for short-term solar irradiance forecasting in a tropical environment” by Frederik Kurzrock et al.
}

\section{David Ham}

david.ham@imperial.ac.uk

Received and published: 29 April 2019

Thank you for specifying exactly which versions of WRF and DART you are using, and pointing to their archival locations. However currently not all the code and data required to run the simulations in the paper is so clearly identified. The input configuration files required to run the simulations are not currently available, and the exact forcing data used in each of those cases is not clear. Please, therefore, archive and reference these configuration files and any pre- or post- processing scripts required to run your experiments or produce the results presented. Note that it is not necessary (and often

Printer-friendly version

Discussion paper 
not possible) to duplicate all the input data, providing configuration files and specifically identifying input data is sufficient.

The configuration files and scripts should be publicly archived in a persistent archive. Many GMD authors find Zenodo a good choice for this, but other archives are available.

\section{Regards,}

David Ham GMD Executive editor.

Interactive comment on Geosci. Model Dev. Discuss., https://doi.org/10.5194/gmd-2019-29, 2019. 